\title{
POLYNOMIAL-TIME IDENTIFICATION OF ROBUST NETWORK FLOWS UNDER UNCERTAIN ARC FAILURES
}

\author{
VLADIMIR L. BOGINSKI, CLAYTON W. COMMANDER, AND TIMOFEY TURKO
}

\begin{abstract}
We propose LP-based solution methods for network flow problems subject to multiple uncertain arc failures, which allow finding robust optimal solutions in polynomial time under certain conditions. We justify this fact by proving that for the considered class of problems under uncertainty with linear loss functions, the number of entities in the corresponding LP formulations is polynomial with respect to the number of arcs in the network. The proposed formulation is efficient for sparse networks, as well as for timecritical networked systems, where quick and robust decisions play a crucial role.
\end{abstract}

\section{INTRODUCTION AND OUTLINE}

Network flow problems are among the most popular topics in operations research with numerous papers and textbooks devoted to their study [1, 3, 9, 15, 18]. These problems have a wide variety of important applications in many areas. Although deterministic formulations of these problems are well-studied, in many practical situations one has to deal with uncertainties associated with possible failures of network components (e.g., each arc has a probability of failure). Formulations and optimal solutions of these problems need to take into account these uncertainty factors.

The main difficulty arising in addressing these issues is the drastic increase in the computational complexity of the resulting optimization problems. For example, Corea and Kulkarni [7] have considered the MINIMUM COST FLOW PROBLEM where the length (cost) of each edge is a random variable. They construct continuous time Markov chains to derive stable algorithms computing the distribution of the minimum cost. The downside to this method is that the state space grows exponentially with the size of the graph, leading to computational difficulties when attempting to solve large-scale instances. Glockner and Nemhauser [12] have also considered dynamic network flow problems where the arc capacities are random variables. They describe novel decomposition techniques for the resulting multi-stage stochastic linear program. Doulliez and Rao [10] have studied the problem of determining the maximum flow in a graph subject to a single arc failure. Along this line, Aneja et al. [2] considered the MAXIMUM FLOW PROBLEM and present a strongly polynomial algorithm for maximizing the residual flow in a graph after a single arc is destroyed. Cormican et al. [8] have formulated stochastic programming formulations of the NETWORK INTERDICTION PROBLEM in which arcs are removed in order to minimize the expected maximum flow on the network. These problems are modeled as two-stage stochastic programming problems and are $\mathcal{N} \mathcal{P}$-hard. On the other hand, Bertsimas and Sim [4] proposed polynomial-time algorithms for solving robust versions of certain classes of optimization problems, including network flows, which are based on solving a polynomial number of polynomially solvable problem instances. It should be noted that the term robust optimization is often used to denote the class of problems in which the worst possible outcomes in terms of losses in a system are taken into account. These losses can be 
minimized and/or restricted in the corresponding optimization problems, which results in "robust" solutions.

In this paper, we use the approach of restricting the conditional expectation of the worstcase possible losses of flow in a network and propose Linear Programming (LP) based methods for finding robust solutions of network flow problems under uncertainty. In particular, we provide a polynomially solvable formulation for the ROBUST MINIMUM COST FLOW PROBLEM (RMCF), in which each arc in a network has a probability of failure. This method assumes that all the decisions regarding assigning the flows to the network are made at the initial time moment. The attractive feature of this formulation is that it allows one to find a robust solution for a given problem instance by solving one LP problem with a polynomial number of variables and constraints. In addition, the proposed formulation allows one to adjust certain parameters of the model to control the tradeoffs between the cost efficiency and the robustness of the solution. Therefore, the proposed formulation is useful in practical situations where time-critical decisions need to be made. To our knowledge, this is the first formulation of this type proposed for network flow problems under uncertainty.

The considered formulations use a quantitative representation of the total collateral loss of flow in a network (referred to as a loss function) under a set of arc failure scenarios. In these settings, we show that for Linear Programming problems under uncertainty with linear loss functions, a polynomial number of scenarios is sufficient to obtain an arbitrarily good approximation of the true conditional mean of the distribution of the loss function, which corresponds to a certain percentage of the worst-case losses. Specifically, we prove that this number is polynomial with respect to the number of arcs (edges) in the network. This result has direct impact on the computational complexity of the considered problem. In the proposed setup, it leads to a robust LP problem with the polynomial number of entities, which makes the robust version of the original problem polynomially solvable.

It should be noted that this approach is especially promising for sparse networks (with a relatively low number of edges), which are very common in real-life applications. Moreover, this polynomial-time procedure will be applicable in many areas where network flows play a crucial role, especially in time-critical systems (e.g., in military settings) where efficient and robust decisions need to be made at the initial time moment as quickly as possible. Specific examples include routing information in a rapidly deployable tactical data network, and maximizing traffic flows along highways subject to extreme congestion, effectively causing an arc failure. Routing of vehicles in battlefield scenarios is particularly applicable since the probability of a road being "out of service" due to an ongoing fire fight, or other disturbance renders flow along this arc infeasible.

\section{Statistical Measures of Losses for Optimization Problems Under UNCERTAINTY}

To facilitate the upcoming discussion, we briefly review some of the basic statistical concepts (commonly referred to as quantitative risk measures), which we utilize in this study.

One of the most well-known risk measures used in robust optimization under uncertainty is known as Value-at-Risk (VaR) [13]. VaR provides an upper bound, or percentile on a given loss distribution. For example, consider an application in which a constraint must be satisfied within a specific confidence level $\alpha \in(0,1]$. Then the corresponding $\alpha$-VaR value is the lowest value $\zeta$ such that with probability $\alpha$, the loss does not exceed $\zeta$ [14]. In economic terms, VaR is simply the maximum amount at risk to be lost from an investment. 


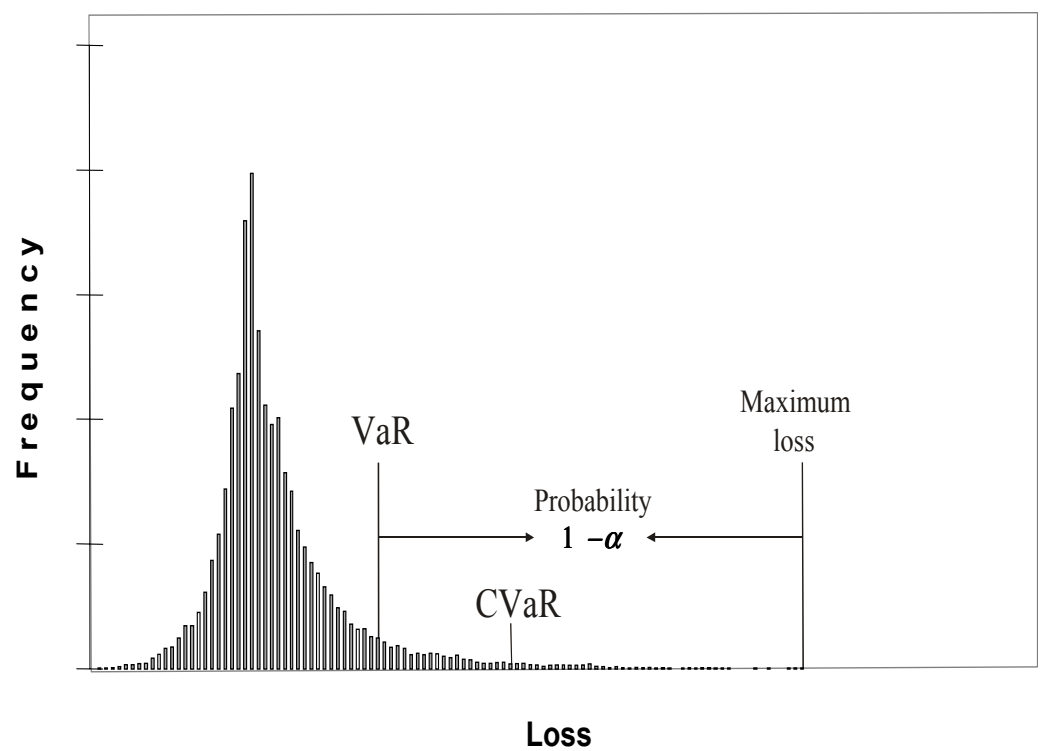

FIGURE 1. A graphical depiction of VaR and CVaR.

VaR is the most widely applied risk measure in probabilistic settings primarily because it is conceptually simple and easy to incorporate into a mathematical model [6]. However with this ease of use is accompanied by several complicating factors. Some disadvantages are that the inclusion of VaR constraints increases the number of discrete variables in a problem. Thus a polynomially solvable problem is likely to become $\mathcal{N} \mathcal{P}$-hard [11] after the VaR constraints are added to the model. Also, VaR is not a so-called coherent risk measure, implying among other things that it is non-convex and not sub-additive.

Another risk measure closely related to $\mathrm{VaR}$ is the so-called Conditional Value-at-Risk (CVaR) (also known in the finance literature as Expected Tail Loss, Expected Shortfall, and Worst Conditional Expectation). By definition, CVaR is the conditional expectation of the loss under the condition that VaR is exceeded. Clearly, CVaR is a more conservative measure of risk than VaR. Rockafellar and Uryasev [16] proved several important results regarding optimization of $\mathrm{CVaR}$, which make this risk measure rather attractive from the optimization viewpoint. In particular, CVaR has been shown to possess the properties that VaR lacks; in particular, it is coherent (which includes convexity among other properties). This makes this statistical measure much more convenient to handle in optimization models. A simple illustrative graphical representation of the relationship between CVaR and VaR is shown in Figure 1 .

In order to define VaR and CVaR more formally, we introduce the following notations. Suppose that the random variable $L(x, y)$ (referred to as a loss function) represents a total collateral loss associated with a decision vector $x \in X \subseteq \mathbb{R}^{n}$, and a random vector $y \in \mathbb{R}^{m}$ which represents the uncertain parameters that may affect the performance of a system under consideration. Assume that $y$ is governed by a probability measure $P$ on a Borel set, say $Y$ [6]. Then the loss $L(x, y)$ for each $x \in X$ is a random variable having a distribution in $\mathbb{R}$ induced by that of $y$. Therefore the probability of $L(x, y)$ not exceeding 
some value $\zeta$ is defined as

$$
\psi(x, \zeta):=P\{y \mid L(x, y) \leq \zeta\} .
$$

By fixing $x$, the cumulative distribution function of the loss associated with the decision $x$ is thus given by $\psi(x, \zeta)[19]$.

Given the loss random variable $L(x, y)$ and any $\alpha \in(0,1)$, we can use equation (1) to define $\alpha$-VaR as

$$
\zeta_{\alpha}(x):=\min \{\zeta \in \mathbb{R}: \psi(x, \zeta) \geq \alpha\} .
$$

From this we see that the probability that the loss $L(x, y)$ exceeds $\zeta_{\alpha}(x)$ is $1-\alpha$. Using the definition above, CVaR is the conditional expectation that the loss according to the decision vector $x$ dominates $\zeta_{\alpha}(x)$ [16]. Thus we have $\alpha$-CVaR denoted as $\phi_{\alpha}(x)$ defined as

$$
\phi_{\alpha}(x):=\mathbf{E}\left\{L(x, y) \mid L(x, y) \geq \zeta_{\alpha}(x)\right\} .
$$

In order to include $\mathrm{CVaR}$ and $\mathrm{VaR}$ constraints in optimization models, one can characterize $\zeta_{\alpha}(x)$ and $\phi_{\alpha}(x)$ in terms of a function $F_{\alpha}: X \times \mathbb{R} \mapsto \mathbb{R}$ defined by

$$
F_{\alpha}(x, \zeta):=\zeta+\frac{1}{(1-\alpha)} \mathbf{E}\{\max \{L(x, y)-\zeta, 0\}\} .
$$

It can be shown that as a function of $\zeta, F_{\alpha}(x, \zeta)$ is convex and continuously differentiable [16]. Moreover, for any $x \in X, \phi_{\alpha}(x)=\min _{\zeta \in \mathbb{R}} F_{\alpha}(x, \zeta)$, for all $\zeta \in \mathbb{R}$. Furthermore, if $A_{\alpha}(x):=\operatorname{argmin}_{\zeta \in \mathbb{R}} F_{\alpha}(x, \zeta)$ is the set consisting of the values of $\zeta$ for which $F$ is minimized, then $A_{\alpha}(x)$ is a non-empty, closed and bounded interval and $\zeta_{\alpha}(x)$ is the left endpoint of $A_{\alpha}(x)$. In particular, it is always the case that $\zeta_{\alpha}(x) \in \operatorname{argmin}_{\zeta \in \mathbb{R}} F_{\alpha}(x, \zeta)$ and $\psi_{\alpha}(x)=F_{\alpha}\left(x, \zeta_{\alpha}(x)\right)$ [16]. It has also been shown that for any probability threshold $\alpha$ and loss tolerance $C$, constraining $\phi_{\alpha}(x) \leq C$ is equivalent to constraining $F_{\alpha}(x, \zeta) \leq$ $C$ [17.

\section{Robust Minimum Cost Flow Problem Under UnCERTAinty With CVAR CONSTRAINTS}

In general, an instance of the MINIMUM COST FLOW PROBLEM (MCF) consists of a directed graph $G=(V, E)$, where each edge $(\operatorname{arc})(i, j) \in E$ has an associated $\operatorname{cost} c_{i j}$ per unit of flow along this edge, as well as a capacity $u_{i j}$ denoting the maximum amount of flow that can traverse edge $(i, j)$. For each node $i \in V, d_{i}$ denotes the demand (supply) of node $i$. Then the MCF can be formulated as the following well-known linear program:

$$
\begin{array}{ll}
\text { (MCF) } \min & \sum_{(i, j) \in E} c_{i j} x_{i j} \\
\text { s.t. } & \sum_{\{j:(i, j) \in E\}} x_{i j}-\sum_{\{j:(j, i) \in E\}} x_{j i}=d_{i}, \forall i \in V, \\
& 0 \leq x_{i j} \leq u_{i j}, \forall i, j \in V .
\end{array}
$$

Next, suppose that $L(x, y)$ is a loss function, where $x$ is a vector of decision variables and $y$ is a vector of uncertain parameters. In the context of a network flow problem, $x_{i j}$ represents the amount of flow through arc $(i, j)$. Further, let $y$ be a random vector representing the uncertain arc failures in the network. Each component of this vector $y_{i j}$ is a Bernoulli random variable defined as follows: 


$$
y_{i j}:=\left\{\begin{array}{l}
1, \text { with probability } p_{i j} \\
0, \text { with probability } 1-p_{i j}
\end{array}\right.
$$

where $p_{i j}$ is the probability of failure for the arc $(i, j)$.

Furthermore, based on the distributions of $y_{i j}$, one can generate a set of failure scenarios $s=1,2, \ldots, S$, where the values $y_{i j}^{s}$ are defined for each scenario as follows:

$$
y_{i j}^{s}:=\left\{\begin{array}{l}
1, \text { if } \operatorname{arc}(i, j) \text { fails under scenario } s \\
0, \text { otherwise }
\end{array}\right.
$$

Then, the random variable $L(x, y)$ representing the total loss can be defined as

$$
L(x, y)=\sum_{(i, j) \in E} \lambda_{i j} x_{i j} y_{i j}
$$

Note that in this definition we introduce the coefficients $\lambda_{i j}$ corresponding to each arc $(i, j)$. They can be viewed as arc importance coefficients. The values of these coefficients may differ depending on specific applications. In many practical situations, the relative importance of certain arcs may be different from a decision maker's viewpoint; therefore, these "penalty coefficients" provide a certain degree of flexibility for the quantitative expression of the collateral losses associated with the failures of different arcs. In the further discussion, without loss of generality, we set $\lambda_{i j}=1 \forall(i, j) \in E$; however, all the formulas derived below are valid for arbitrary values of $\lambda_{i j}$.

Further, using the above notations, we define the realization of the random variable $L(x, y)$ for each scenario $s=1, \ldots, S$ :

$$
L\left(x, y^{s}\right)=\sum_{(i, j) \in E} x_{i j} y_{i j}^{s}
$$

Then with this loss function, the characteristic function for CVaR constraints can be approximated by

$$
\bar{F}_{\alpha}(x, \zeta)=\zeta+\frac{1}{1-\alpha} \sum_{s=1}^{S} \pi_{s} \max \left\{\sum_{(i, j) \in E} x_{i j} y_{i j}^{s}-\zeta, 0\right\}
$$

where $\pi_{s}$ is the probability of scenario $y^{s}$, for $s=1,2, \ldots, S$, [16]. Since $L(x, y)$ is linear with respect to $x, \bar{F}_{\alpha}(x, \zeta)$ is convex and piecewise linear [14]. If we assume that each scenario is equally likely, that is $\pi_{s}=\frac{1}{S}, \forall s=1,2, \ldots, S$, then (11) reduces to

$$
\bar{F}_{\alpha}(x, \zeta)=\zeta+\frac{1}{S(1-\alpha)} \sum_{s=1}^{S} \max \left\{\sum_{(i, j) \in E} x_{i j} y_{i j}^{s}-\zeta, 0\right\}
$$


Then we can model the robust MCF problem with CVaR constraints as

$$
\begin{array}{ll}
\text { (RMCF-1) } & \text { Minimize } \sum_{\{(i, j) \in E\}} c_{i j} x_{i j} \\
& \text { s.t. } \\
& \sum_{\{j:(i, j) \in E\}} x_{i j}-\sum_{\{j:(j, i) \in E\}} x_{j i}=d_{i}, \forall i \in V, \\
& 0 \leq x_{i j} \leq u_{i j}, \forall(i, j) \in E, \\
& \zeta+\frac{1}{S(1-\alpha)} \sum_{s=1}^{S} \max \left\{\sum_{(i, j) \in E} x_{i j} y_{i j}^{s}-\zeta, 0\right\} \leq C, \\
& \zeta \in \mathbb{R} .
\end{array}
$$

We can linearize $\bar{F}_{\alpha}(x, \zeta)$ by using a set of extra variables $t_{s}, s=1,2, \ldots, S$, and replacing $\bar{F}_{\alpha}(x, \zeta)$ by the linear function $\zeta+\frac{1}{S(1-\alpha)} \sum_{s=1}^{S} t_{s}$ and adding the set of linear constraints

$$
\begin{aligned}
& t_{s} \geq L\left(x, y^{s}\right)-\zeta, \forall s=1,2, \ldots, S \\
& t_{s} \geq 0, \forall s=1,2, \ldots, S .
\end{aligned}
$$

Thus, the fully linearized mathematical programming formulation of the ROBUST MINIMUM COST FLOW PROBLEM with Conditional Value-at-Risk constraints is given as

$$
\begin{array}{ll}
\text { (RMCF-LP) } & \text { Minimize } \sum_{(i, j) \in E} c_{i j} x_{i j} \\
& \sum_{\{j:(i, j) \in E\}} x_{i j}-\sum_{\{j:(j, i) \in E\}} x_{j i}=d_{i}, \forall i \in V, \\
& \zeta+\frac{1}{S(1-\alpha)} \sum_{i=1}^{S} t_{s} \leq C, \\
& t_{s} \geq \sum_{(i, j) \in E} x_{i j} y_{i j}^{s}-\zeta, \forall s=1,2, \ldots, S, \\
& t_{s} \geq 0, \forall s=1,2, \ldots, S, \\
& \zeta \in \mathbb{R}, \\
& 0 \leq x_{i j} \leq u_{i j}, \forall(i, j) \in E .
\end{array}
$$

Further, note that the mass-balance constraints in (6) from formulation MCF may be relaxed in formulation RMCF-LP. This modification can be made in order to address possible solution infeasibility issues that may arise due to the additional restrictions on the robustness of the solution. In the modified formulation, the penalty terms $h_{i}$ representing the possible loss of flow (e.g., unsatisfied demand) at node $i$ can added to the objective function and the corresponding constraints. Clearly, it is desired that the penalty variables $h_{i}$ are equal to zero in the optimal solution, which can be modeled by a standard technique of multiplying these variables by "sufficiently large" coefficients $M_{i}$ in the objective. Then the objective and the mass-balance constraints in the above formulation may be rewritten as 


$$
\sum_{(i, j) \in E} c_{i j} x_{i j}+\sum_{i \in V} M_{i} h_{i}
$$

and

$$
\sum_{\{j:(i, j) \in E\}} x_{i j}-\sum_{\{j:(j, i) \in E\}} x_{j i}=d_{i}-h_{i}, \forall i \in V
$$

It is worth mentioning that the proposed formulation allows one to adjust certain parameters of the model (e.g., the probability level $\alpha$ and the parameter $C$ in the CVaR constraint) to control the tradeoffs between the cost efficiency and the robustness of the solution. Moreover, as indicated above, application specific penalty coefficients can also be introduced in the model to add even more degrees of flexibility in this respect. Also, the definition of "sufficiently large" in terms of the coefficients $M_{i}$ is dependent upon the problem instance and requires some judgement for implementation.

Further, note that this LP formulation contains $O(|V|+|E|+S)$ variables and $O(|V|+$ $|E|+S)$ constraints. Therefore, the next subject of our interest is how large the number of scenarios $S$ needs to be to ensure sufficiently good statistical properties of the optimal robust solutions. In the next section, we address this issue and identify the conditions under which $S$ is polynomial with respect to $|E|$ (and $|V|$ ), which makes the proposed problem formulation polynomially solvable.

\section{Computational Complexity and Polynomial Solvability Issues}

In this section, we discuss the issues of obtaining a "good" approximation of the true distribution of the losses in the above formulations. More specifically, we investigate the issue of the required number of scenarios that would ensure that the true value of the CVaR function $F_{\alpha}(x, \zeta)$ is close enough to the sample (scenario-based) value that was used in the aforementioned formulation with a high confidence level. In particular, the subject of special interest would be the dependency of the required number of scenarios on the input size (i.e., the size of the network). This is especially important as the number of scenarios considered will directly affect the number of additional constraints in the model. In particular, if the required number of scenarios needed is exponential in the size of the input, then the polynomial solvability of the problem is undermined by the exponential number of constraints. In this section, we prove that the required number of samples needed to accurately approximate $\mathrm{CVaR}$ function corresponding to linear loss functions is in fact polynomial in the size of the input (e.g., the number of nodes and edges in the network) under certain conditions.

For the discussion below, let the sample (scenario-based) mean of each random parameter $y_{i j}$ be defined as $\bar{y}_{i j}:=\frac{1}{S} \sum_{s=1}^{S} y_{i j}^{s}$. Also, assume that all random variables $y_{i j}$ are independent with a finite mean $\mu_{i j}$ and a finite variance $\sigma_{i j}^{2}$ (which is true for the considered case with Bernoulli random variables). Note that in the considered case $\mu_{i j}=p_{i j} \in[0,1]$ and $\sigma_{i j}^{2}=p_{i j}\left(1-p_{i j}\right) \in[0,1], \forall(i, j) \in E$. With this, we have the following proposition.

Proposition 1. Consider a loss function which is linear with respect to the uncertain parameters such as the one defined in Equation (10), along with its corresponding approximation of the characteristic CVaR function $\bar{F}_{\alpha}(x, \zeta)$ (see Equation (12)). Then for any fixed $\varepsilon, \beta \in \mathbb{R}$ such that $\varepsilon>0$, and $\beta \in(0,1]$, the required number of scenarios $S$ to guarantee that $P\left(\left|\bar{F}_{\alpha}(x, \zeta)-F_{\alpha}(x, \zeta)\right|<\varepsilon\right)>1-\beta$ is $\mathcal{O}\left(m^{2} / \varepsilon^{2}\right)$, where $m=|E|$ is the number of arcs in the original network. 
Proof. For simplicity, let

$$
r_{s}:=\max \left\{\sum_{(i, j) \in E} x_{i j} y_{i j}^{s}-\zeta, 0\right\} .
$$

Then in their full forms, we have

$$
F_{\alpha}(x, \zeta)=\zeta+\frac{1}{(1-\alpha)} \mathbf{E}\left\{r_{s}\right\}
$$

and

$$
\bar{F}_{\alpha}(x, \zeta)=\zeta+\frac{1}{S(1-\alpha)} \sum_{s=1}^{S} r_{s}
$$

In order to prove the proposition, it is enough to show that the required number of scenarios to guarantee that for any $\varepsilon, \beta \in \mathbb{R}$ such that $\varepsilon>0$, and $\beta \in(0,1], P\left(\left|\frac{1}{S} \sum_{s=1}^{S}\left[r_{s}-\mathbf{E}\left\{r_{s}\right\}\right]\right|<\varepsilon\right)>$ $1-\beta$ is $\mathcal{O}\left(m^{2}\right)$. Notice that for all $s=1,2, \ldots, S, r_{s}$ have the same distribution. Therefore, we can denote their variances as $\operatorname{Var}\left(r_{s}\right) \equiv \operatorname{Var}(r), \forall s$. Next recall that for any random variable $X, \operatorname{Var}(\max \{X, 0\}) \leq \operatorname{Var}(X)$. Thus it follows that

$$
\operatorname{Var}(r) \leq \operatorname{Var}\left(\sum_{(i, j) \in E} x_{i j} y_{i j}\right)=\sum_{(i, j) \in E} x_{i j}^{2} \operatorname{Var}\left(y_{i j}\right) \leq U^{2} m,
$$

where $U:=\max _{i, j}\left\{x_{i j}\right\}$ (clearly, $U$ will be finite assuming that all supplies and demands in the network are finite), and $\operatorname{Var}\left(y_{i j}\right)=\sigma_{i j}^{2} \leq 1$, as indicated above. Let $\Phi_{0,1}(z)$ be the cumulative distribution function of a standard normal random variable, and $\bar{\Phi}_{0,1}(z)=$ $1-\Phi_{0,1}(z)$. Then applying the basic standardization technique [5], we see that

$$
P\left(\left|\frac{1}{S} \sum_{s=1}^{S}\left[r_{s}-\mathbf{E} r_{s}\right]\right| \leq \varepsilon\right)=P\left(\left|\frac{\sum_{s=1}^{S} r_{s}-S \mathbf{E} r_{s}}{\operatorname{Var}(r) \sqrt{S}}\right| \leq \frac{\sqrt{S} \varepsilon}{\operatorname{Var}(r)}\right) \rightarrow 1-2 \bar{\Phi}_{0,1}\left(\frac{\sqrt{S} \varepsilon}{\operatorname{Var}(r)}\right),
$$

as $S \rightarrow \infty$. Now, it follows that if

$$
1-2 \bar{\Phi}_{0,1}\left(\frac{\sqrt{S} \varepsilon}{\operatorname{Var}(r)}\right)=1-\beta
$$

then

$$
\frac{\sqrt{S} \varepsilon}{\operatorname{Var}(r)}=c_{\beta / 2},
$$

where the notation $c_{\beta}$ represents the $(1-\beta)$-quantile of the standard normal distribution (which is a fixed finite number for any fixed $\beta$ ). From here we see that the sufficient number of scenarios is

$$
S=\left(\frac{c_{\beta / 2} \operatorname{Var}(r)}{\varepsilon}\right)^{2}=O\left([\operatorname{Var}(r)]^{2} / \varepsilon^{2}\right)=O\left(m^{2} / \varepsilon^{2}\right) .
$$

Furthermore, for any fixed $\varepsilon, S=\mathcal{O}\left(m^{2}\right)$.

Proposition 1 provides the justification for the use of Conditional Value-at-Risk constraints for network flow problems in terms of computational complexity and efficiency. That is, by incorporating CVaR constraints we are able to guarantee that the sample approximation of the CVaR function can be made arbitrarily accurate while the size of the 
problem remains polynomial with respect to the input. Thus we can find robust solutions to these problems in polynomial time using standard Linear Programming techniques.

\section{ILlustratiVE EXAMPLE}

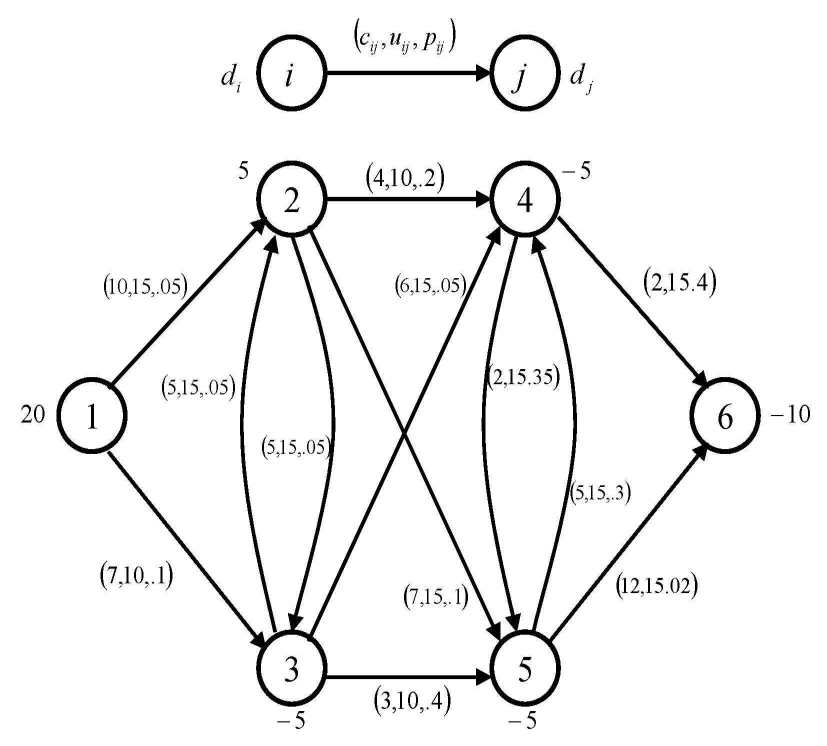

(a) The original network.

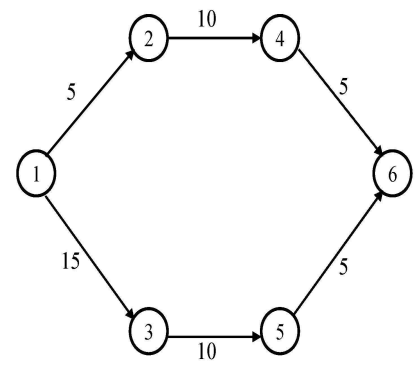

(b) The ideal optimal solution. The objective value is 295 .

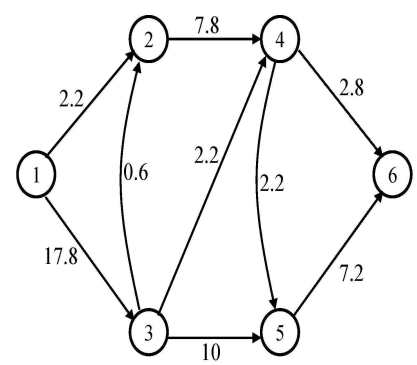

(c) The optimal solution with CVaR. In this example, $S=100$ and the objective value is 340.2 .

FIGURE 2. The min-cost flow problem is solved for the graph in (a). $c_{i j}, u_{i j}$, and $p_{i, j}$ represent the cost, maximum capacity, and probability of failure for arc $(i, j)$ respectively.

In this section, we present an illustrative numerical example which demonstrates how the proposed approach can change the deterministic optimal flow assignments when uncertain failures of network edges are introduced. Consider Figure 2. Suppose we are solving 
the MINIMUM COST FLOW PROBLEM where each edge has a given probability of failure. Subfigure 2(a) represents the original network and the triplets $\left(c_{i j}, u_{i j}, p_{i j}\right)$, correspond to the cost, maximum capacity, and probability of failure for each arc $(i, j)$.

In Subfigure 2(b), the optimal ideal solution (without the failures) is shown. The ideal case represents the deterministic MCF problem, or equivalently, the probabilistic version when all edge failure probabilities are 0 . The optimal solution (minimum cost) for this instance is 295 . However by taking into account the edge failure probabilities, we see that this is not the optimal solution in this case. In 2(c), the optimal solution is shown with CVaR constraints. In this example, the total loss in the worst $10 \%$ of scenarios is constrained to be less than 0.23 units of flow. That is, in formulation RMCF-LP, $\alpha=0.9$ and $C=0.23$. We generated 100 scenarios uniformly at random for this example, with up to $20 \%$ of the arcs failing in any scenario. These are arbitrary values and can be specified by the user as the situation calls. In this case, the objective function value is 340.2 (obviously larger than the one for the ideal case). We see that in fact, the solution tends to push more flow over arcs with lower probabilities of failure accepting the higher cost for the "hedging". This is exactly the behavior we would predict. An interpretation is that by diversifying the flow across various arcs, we ensure that the solution is more robust in the event of (multiple) arc failures.

Notice that in the above example, the flows along each arc no longer integral after the incorporation of the CVaR constraints. This follows since the constraint matrix in the RMCF-LP formulation is no longer unimodular. However, the utility of the proposed approach is not lost. For example, consider the application of flow routing on an information exchange network in which the flow values represent megabytes of data to be sent along each arc. Then non-integral values such as those obtained above correspond to realizable flows.

\section{CONCLUSIONS}

In this paper, we proposed polynomial-time Linear Programming based solution approaches for network flow problems subject to uncertain multiple arc failures. Further, we have shown that for linear loss functions, the considered LP formulations would contain a polynomial number of entities. An illustrative example was given to demonstrate a typical performance of the proposed procedure in the context of the MINIMUM COST FLOW PROBLEM under uncertain arc failures. In addition, the proposed formulations allow one to adjust certain parameters of the model to control the tradeoffs between the cost efficiency and the robustness of the model. The proposed models have a wide variety of real-world applications, especially in time-critical systems and sparse networks in both military and civilian settings.

\section{REFERENCES}

[1] R.K. Ahuja, T.L. Magnanti, and J.B. Orlin. Network Flows: Theory, Algorithms, and Applications. PrenticeHall, 1993.

[2] Y.P. Aneja, R. Chandrasekaran, and K.P.K. Nair. Maximizing residual flow under an arc destrucion. Networks, 38(4):194-198, 2001.

[3] D.P. Bertsekas. Network Optimization - Continuous and Discrete Models. Athena Scientific, Belmont, Massachusetts, 1998.

[4] D. Bertsimas and M. Sim. Robust discrete optimization and network flows. Mathematical Programming, 98:49-71, 2003

[5] G. Casella and R.L. Berger. Statistical Inference. Duxbury, 2 edition, 2002.

[6] C.W. Commander, P.M. Pardalos, V. Ryabchenko, and S. Uryasev. The wireless network jamming problem. Journal of Combinatorial Optimization, 14(4):481-498, 2007. 
[7] G.A. Corea and V.G. Kulkarni. Minimum cost routing on stochastic networks. Operations Research, 38(3):527-536, 1990.

[8] K.J. Cormican, D.P. Morton, and R.K. Wood. Stochastic network interdiction. Operations Research, 46(2):184-197, 1998.

[9] G.B. Dantzig. Linear Programming and Extensions. Princeton University Press, 1963.

[10] P.J. Doulliez and M.R. Rao. Maximal flow in a multi-terminal network with any one arc subject to failure. Management Science, 18(1):48-58, 1971.

[11] M.R. Garey and D.S. Johnson. Computers and Intractability: A Guide to the Theory of NP-Completeness. W.H. Freeman and Company, 1979.

[12] G.D. Glockner and G.L. Nemhauser. A dynamic network flow problem with uncertain arc capacities: Formulation and problem structure. Operations Research, 48(2):233-242, 2000.

[13] G. Holton. Value-at-Risk: Theory and Practice. Academic Press, 2003.

[14] P. Krokhmal, J. Palmquist, and S. Uryasev. Portfolio optimization with conditional value-at-risk objective and constraints. The Journal of Risk, 4(2):11-27, 2002.

[15] R.T. Rockafellar. Network Flows and Monotropic Programming. John Wiley \& Sons, New York, 1984.

[16] R.T. Rockafellar and S. Uryasev. Optimization of conditional value-at-risk. The Journal of Risk, 2(3):21-41, 2000.

[17] R.T. Rockafellar and S. Uryasev. Conditional value-at-risk for general loss distributions. Journal of Banking and Finance, 26:1443-1471, 2002.

[18] A. Schrijver. On the history of the transportation and maximum flow problem. Mathematical Programming, 91:437-445, 2002.

[19] S. Uryasev. Conditional value-at-risk: Optimization algorithms and applications. Financial Engineering News, 14:1-5, 2000.

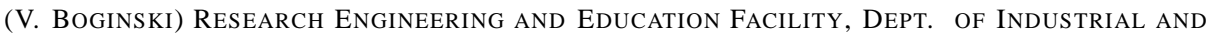
SYSTEMS ENGINEERING, UNIVERSITY OF FLORIDA, SHALIMAR, FL, USA.

E-mail address: boginski@reef.ufl.edu

(C.W. Commander) Air Force Research Laboratory, Munitions Directorate, Eglin Air FORCE BASE, FL, USA.

E-mail address: clayton.commandereeglin.af.mil

(T. TURKo) DEPT. OF INDUSTRIAL AND SYSTEMS ENGINEERING, UNIVERSITY OF FLORIDA, GAINES VILLE, FL, USA.

E-mail address: tturko@ufl.edu 